\title{
Worksharing, access and bypass: the structure of prices in the postal sector ${ }^{1}$
}

\author{
Etienne Billette de Villemeur ${ }^{2}$ \\ University of Toulouse (IDEI and GREMAQ) \\ 21 Allée de Brienne \\ 31000 Toulouse \\ Helmuth Cremer, \\ University of Toulouse (IDEI and GREMAQ) \\ 21 Allée de Brienne \\ 31000 Toulouse \\ Bernard Roy ${ }^{3}$ \\ La Poste \\ 44 Bvd Vaugirard \\ 75757 Paris Cedex 15 \\ and \\ Joëlle Toledano \\ SUPELEC \\ Plateau du Moulon \\ 3 , rue Joliot Curie \\ Gif-sur-Yvette 91192 Cedex
}

May 2005, revised October 2006

\footnotetext{
${ }^{1}$ This paper has been presented at 13th CRRI Conference on Postal and Delivery Economics, June 2005. We thank all the participants and, in particular, Robert Mitchell and John Panzar for their comments. We thank the referees and the editor for their detailed and constructive comments that were of tremendous use to us.

${ }^{2}$ Corresponding author; e-mail: etienne.devillemeur@univ-tlse1.fr.

${ }^{3}$ The views expressed in this paper are those of the authors and do not necessarily reflect the views of La Poste.
} 


\begin{abstract}
This paper studies a liberalized postal market where entrants may offer end-to-end products or concentrate on one of the segments of the network. Absent effective bypass, entry does not appear to be a serious financial threat to the incumbent, even when the products are perfect substitutes. This is no longer true when the entrant offers cheaper service in delivery. Then, the universal service provider may loose the entire pre-sorted mail market. It is left with probably low volume demand from households and from firms with high preparation cost, and its financial viability may be jeopardized.
\end{abstract}

JEL classification: L51; L87; L32

Keywords: postal sector, liberalization, universal service, access, bypass. 


\section{Introduction}

In most network industries, the ongoing liberalization process has spurred an intense debate on the phenomenon of "downstream access". Accordingly this subject has been extensively studied in the economics literature. ${ }^{1}$ In the postal sector the issue of "access" has been relevant long before the debate on liberalization was launched. However, it has appeared under a different form, namely worksharing. Processing workshared mail at a discounted rate is effectively like providing the client with access to one or several segments of the postal network. As in the case of downstream access, we have a situation where the postal operator sells some products which use only part of its network, while other products use the entire network. Put differently, the postal sector has the specific feature that access is a relevant issue even when there are no competing operators in the market. This is reflected in the existing literature on worksharing which typically considers a monopolistic sector; see Billette de Villemeur et al. $(2002,2003 \mathrm{a}){ }^{2}{ }^{2}$

The structure of prices derived in this literature has to be reconsidered when the market opens. Competition may take two different forms. First, once entry has occurred, the demand for workshared mail may in part emanate from the competing operators. Specifically, the entrant may have all or part of its mail delivered by the incumbent (for an access charge) rather then build its own delivery network. In that case competition is limited to the upstream segments (mail preparation, etc.). Consequently, "high" access charges (ECPR type corrected by Ramsey elements) are likely to be optimal. They ensure that the entrant's products contribute in an adequate way to the incumbent's fixed cost (and the financing of the universal service obligation). Second, the entrant may find it optimal to "bypass" the incumbent's network in some areas by setting up its own delivery network. Now competition affects all segments and the ability of the incumbent to cover its fixed costs may be undermined. The possibility of bypass limits the potential for the entrant's products to contribute towards universal service costs and pricing rules will have to be amended. The incumbent's pricing policy will be modified accordingly with the expection of a rather significant impact.

\footnotetext{
${ }^{1}$ See Laffont and Tirole $(1996,2000)$ and Armstrong (2002).

${ }^{2}$ See also Crew and Kleindorfer (1995), Mitchel (1999), Sherman (2001) and Panzar (2002).
} 
In this paper, we put together a number of building blocks that we have developed over the last years. Specifically, we build on Billette de Villemeur et al. (2003a, 2004 and 2005) which we generalize by introducing different types of delivery areas and the possibility of bypass. Probably the most useful contribution of this is the specification of the "demand model" 3 which allows business mailers to choose to purchase end-to-end service and/or workshared service from either the incumbent or the entrant. Furthermore, the products of the incumbent and entrant are differentiated. However, the workshared and end-to-end services of both providers are perfect substitutes in terms of their value to final consumers. The closest predecessor to our analysis is Panzar (2005). ${ }^{4}$ He also used a setting based on Billette de Villemeur et al. (2003a) to study the interaction between liberalization and unbundling and to introduce the notion of "piecemeal bypass". While our model is more complex than Panzar's, the two papers are fairly complementary in that they envision different types of entry. ${ }^{5}$ The crucial ingredient in Panzar's model is end-to-end competition (possibly through piecemeal bypass) on single-piece mail. We do not consider competition in the single-piece market segment. Instead, we concentrate on the industrial mail market that appears to be the main theater for competition at least within the European context.

We provide optimal pricing rules for the specification with general demand (but without uniform pricing constraint). Even though we are able to provide some analytical results, it is clear that a fully fledged study of the general model requires numerical simulations. That part of the paper is thus to be understood as an essentially methodological contribution, setting up a framework to be used in future numerical studies. In the second part of the paper we look at a more restrictive setting and provide crisper results that are not only interesting for their own sake but have important policy implications. The main finding from that perspective is that the possibility of bypass may have a rather drastic impact on the results. It brings about not just a quantitative change in the level of prices but a qualitative shift in the very nature of potential

\footnotetext{
${ }^{3}$ We thank the referee for suggesting this terminology.

${ }^{4}$ The current version of Panzar's paper was presented also presented at the 13th CRRI Conference on Postal and Delivery Economics. However, his analysis is anterior to ours. An earlier version of his paper (presented at IDEI in November 2003) already contained the crucial features.

${ }^{5}$ We also provide more elaborated expressions for the pricing rules.
} 
equilibria. Specifically we show that under bypass the universal service operator may not be able to retain the most lucrative market segment which in turn would seriously undermine its financial viability.

\section{Model}

\subsection{Cost and demand}

There are two operators: the incumbent universal service operator $I$ and an entrant $E$. The stylized postal network we consider consists of two segments. Segment 1 corresponds to a composite activity including collecting, sorting and transportation. This activity implies a constant marginal cost of $c_{1}$ for operator $I$ and of $k^{E}$ for operator $E$. Segment 2 is delivery. Operator $I$ 's marginal delivery cost is $c_{2}^{u}$ and $c_{2}^{r}$ in areas $u$ (rban) and $r$ (ural) respectively. Operator $E$ delivers in areas $u$ only, at a marginal cost of $c_{2}^{E}$. In addition, there is a fixed cost associated with the delivery network which differs across areas and operators. The fixed cost of the incumbent in the two areas is given by $F^{u}$ and $F^{r}$ respectively (with $F^{u}<F^{r}$ ). If the entrant decides to build a delivery network in the urban area he incurs a fixed cost of $\Phi^{u}$. The corresponding level for the rural area $\Phi^{r}$ is assumed to be prohibitive (so that the entrant will never find it optimal to deliver in the rural area). ${ }^{6}$ The remaining fixed costs (i.e., those not associated with delivery) are $F$ for operator $I$ and $\Phi$ for operator $E$. There are $N$ addressees of which a fraction $\alpha$ is located in area $u$, while the remaining fraction $(1-\alpha)$ is located in area $r$.

There are two types of senders and two goods. Senders of type $h$ (households) consume good $x$ which is supplied by $I$ and uses both segments. The marginal cost of $x$ is thus given by $c_{1}+c_{2}^{i}, i=u, r$. Customers of type $f$ can patronize the two operators, $I$ and $E$; furthermore they may or may not use segment 1 of the respective operator. When they use the operator's segment, they buy the end-to-end (E2E) product, which is denoted by $x$ for operator $I$ (the product which is also consumed by households) and $y$ for operator $E$. If they do not use segment 1 (thus engaging in worksharing) they consume good $z_{I}$ (incumbent) or $z_{E}$ (entrant). In that case they bear a preparation cost $k$ which is distributed over $[\underline{k}, \bar{k}]$ according to the cumulative distribution $G(k)$

\footnotetext{
${ }^{6}$ To be more precise, the level of fixed cost per addressee in the rural area is supposed to be prohibitive.
} 


\begin{tabular}{l|c|c|c|c}
\multirow{2}{*}{} & \multicolumn{2}{|c|}{$\mathrm{I}$} & \multicolumn{2}{c}{$\mathrm{E}$} \\
\cline { 2 - 5 } & $\mathrm{E} 2 \mathrm{E}$ & Workshared & $\mathrm{E} 2 \mathrm{E}$ & Workshared \\
\hline Notation & $x$ & $z_{I}$ & $y$ & $z_{E}$ \\
Marginal cost operator & $c_{1}+c_{2}$ & $c_{2}$ & $\left(k^{E}+a\right)$ or & $a$ or \\
& & & $\left(k^{E}+c_{2}^{E}\right)$ & $c_{2}^{E}$ \\
Marginal cost customers & $p_{x}$ & $p_{z}+k$ & $q_{y}$ & $q_{z}+k$
\end{tabular}

Table 1: Customers of type $f$ : products and costs

with density $g(k)$. Observe that $k$ arises in addition to the price paid to the operators for $z_{I}$ or $z_{E}$. Except for the cost difference $x$ and $z_{I}$ on the one hand and $y$ and $z_{E}$ on the other hand are considered as perfect substitutes.

Prices are given by $p_{x}, p_{z}^{u}$ and $p_{z}^{r}$ for operator $I$ and by $q_{y}^{u}, q_{y}^{r}, q_{z}^{u}$ and $q_{z}^{r}$ for operator $E$. Observe that $I$ 's single piece mail has a uniform price. All other types of mail may be priced non uniformly (at least in some scenarios).

The Table 1 summarizes the main features of our model. It lists the different products available to type $f$ customers and for each provides the marginal costs for the operators and the marginal cost for the consumer. ${ }^{7}$ It also incorporates some other notations, namely $k^{E}$ and $c_{2}^{E}$ already mentioned (marginal cost of segment 1 and segment 2 for operator $E$ ), as well as a (access charge). Note that the cost for the operator $E$ depends on whether there is access or bypass (see Subsection 2.2 for more details).

Let $S_{h}^{i}\left(x_{h}^{i}\right)$ denote the (gross) surplus of household senders per addressee in area $i=u, r$, while $S_{f}^{i}\left(x_{f}^{i}+z_{I}^{i}, y^{i}+z_{E}^{i}, k\right)$ represents the (gross) surplus of the sender of type $f$ with characteristic $k$, per addressee in area $i=u, r .{ }^{8}$ Observe that $k$ represents not only the preparation cost, but also determines surplus hence demand. ${ }^{9}$ Net surplus is obtained by subtracting total cost: payment to the operator plus cost of activity 1 , if

\footnotetext{
${ }^{7}$ Superscripts $u$ and $r$ are dropped for simplicity.

${ }^{8}$ For simplicity we use surplus as a welfare measure for firms. From a strict welfare economics point of view, this can be understood as representing the surplus of the consumers who buy the goods produced by firms $f$ which use postal services as inputs. One can easily show that our shortcut does not involve any loss of generality in the case where all downstream markets are competitive.

${ }^{9}$ Alternatively one could identify types by, say, $\theta$ and write preparation costs as $k(\theta)$. However, this would just introduce redundant notation.
} 
applicable. The demand function for households is given by:

$$
x_{h}^{i}(p)=\arg \max _{x}\left\{S_{h}^{i}(x)-p x\right\} .
$$

Similarly, the demand functions of the representative sender $f$ are obtained by maximizing net surplus

$$
\begin{aligned}
& S_{f}^{i}\left(x_{f}^{i}+z_{I}^{i}, y^{i}+z_{E}^{i}, k\right)-p_{x} x_{f}^{i}-\left(p_{z}^{i}+k\right) z_{I}^{i}-q_{y}^{i} y^{i}-\left(q_{z}^{i}+k\right) z_{E}^{i} \\
& \text { s.t } \quad x_{f}^{i}, z_{I}^{i}, y_{f}^{i}, z_{E}^{i} \geqslant 0 .
\end{aligned}
$$

This yields four possible cases, labeled (1), (2a), (2b) and (3) with demand functions (for $i=u, r)$ :

(1) $x_{f}^{i}\left(p_{x}, q_{y}^{i}, k\right)$ and $y^{i}\left(p_{x}, q_{y}^{i}, k\right)$

(2a) $z_{I}^{i}\left(p_{z}^{i}+k, q_{y}^{i}, k\right)$ and $y^{i}\left(p_{z}^{i}+k, q_{y}^{i}, k\right)$

(2b) $x_{f}^{i}\left(p_{x}, q_{z}^{i}+k, k\right)$ and $z_{E}^{i}\left(p_{x}, q_{z}^{i}+k, k\right)$

(3) $\quad z_{I}^{i}\left(p_{z}^{i}+k, q_{z}^{i}+k, k\right)$ and $z_{E}^{i}\left(p_{z}^{i}+k, q_{z}^{i}+k, k\right)$ if $p_{z}^{i}+k>p_{x}$ and $q_{z}^{i}+k>q_{y}^{i}$,

if $p_{z}^{i}+k \leq p_{x}$ and $q_{z}^{i}+k>q_{y}^{i}$,

if $p_{z}^{i}+k>p_{x}$ and $q_{z}^{i}+k \leq q_{y}^{i}$

if $p_{z}^{i}+k \leq p_{x}$ and $q_{z}^{i}+k \leq q_{y}^{i}$.

To understand this expression note first that when $p_{z}^{i}+k=p_{x}$ we have $x_{f}^{i}\left(p_{x}, q, k\right)=$ $z_{I}^{i}\left(p_{z}^{i}+k, q, k\right)$ (workshared mail and end to end mail of a given operator are perfect substitutes). Furthermore, all users of type $f$ for which $p_{z}^{i}+k \leq p_{x}$ (i.e., when $\left.k \leq p_{x}-p_{z}^{i}\right)$ find it profitable to buy good $z_{I}^{i}$ at a level $z_{I}^{i}\left(p_{z}^{i}+k, q, k\right)$. Observe that overall per-unit cost of $z^{i}$ is equal to $p_{z}^{i}+k$; it is this overall cost rather than just $p_{z}^{i}$ which determines demand. On the other hand, when $p_{z}^{i}+k>p_{x}$, it is cheaper to consume $x$ (which is otherwise a perfect substitute) and demand is $x_{f}^{i}\left(p_{x}, p_{y}^{i}, k\right)$. Similarly, we have $y^{i}\left(p, q_{y}^{i}, k\right)=z_{E}^{i}\left(p, q_{z}^{i}+k, k\right)$ when $q_{z}^{i}+k=q_{y}^{i}$.

An alternative way to look at this is to define $(i=u, r)$

$$
\widetilde{k}^{i}=p_{x}-p_{z}^{i}, \quad \text { and } \quad \widehat{k}^{i}=q_{y}-q_{z}^{i}
$$

Clearly the relevant cases depend on the comparison between $\widetilde{k}^{i}$ and $\widehat{k}^{i}$. This is illustrated in Figure 1. In Case 1 the customer's level of $k$ is so high that he buys only end-to-end products, from either operator. Case 3 refers to low levels of $k$ where firms engage in worksharing with both operators. Case $2 \mathrm{a}$ and $2 \mathrm{~b}$ are the intermediate cases. In case 2 a the firm buys end-to-end from $E$ while worksharing its mail with $I$. In case 

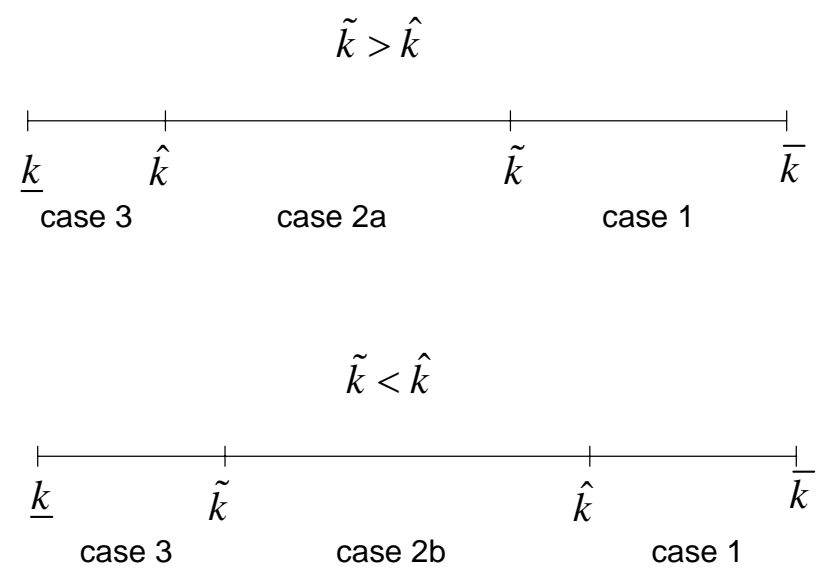

Figure 1: Configuration of type $f$ customers' demand functions.

2b the firm buys end-to-end form $I$ while worksharing its mail with $E$. When $\widetilde{k}^{i}=\widehat{k}^{i}$, only case 1 and 3 are relevant but since prices are endogenous, we cannot impose this restriction on an a priori basis.

Substituting demand functions (1) and (2) into net surplus yields the indirect utility functions $V_{h}^{i}\left(p_{x}\right)$ and $V_{f}^{i}\left(p_{z}^{i}, p_{x}, q_{z}^{i}, q_{y}^{i}, k\right.$ ) (for $f$, these are per addressee in area $i=u, r$ ). ${ }^{10}$ Total utility of type $f$ consumer $k$ is then given by:

$$
\alpha N V_{f}^{u}\left(p_{z}^{u}, p_{x}, q_{z}^{u}, q_{y}^{u}, k\right)+(1-\alpha) N V_{f}^{r}\left(p_{z}^{r}, p_{x}, q_{z}^{r}, q_{y}^{r}, k\right) .
$$

\footnotetext{
${ }^{10}$ For $h$ we have $V_{h}^{i}\left(p_{x}\right)=S_{h}^{i}\left[x_{h}^{i}\left(p_{x}\right)\right]-p_{x} x_{h}^{i}\left(p_{x}\right)$. For $f$, net surplus is given by

$$
S_{f}^{i}\left(x_{f}^{i}+z_{I}^{i}, y^{i}+z_{E}^{i}, k\right)-p_{x} x_{f}^{i}-\left(p_{z}^{i}+k\right) z_{I}^{i}-q_{y}^{i} y^{i}-\left(q_{z}^{i}+k\right) z_{E}^{i} .
$$
}

The full expression for $V_{f}^{i}\left(p_{z}^{i}, p_{x}, q_{z}^{i}, q_{y}^{i}, k\right)$ is complicated because it has to account for the four demand cases. It is omitted for simplicity. 


\subsection{Access, bypass and profit levels}

Operator $E$ pays an access charge of $a^{r}$ per unit of (rural) mail which is to be delivered by $I$. Operator $I$ is assumed not to be allowed to discriminate in the pricing of the downstream services it provides entrants and its own customers, giving rise to the requirement that $a^{r}=p_{z}^{r}$. Consequently $p_{z}^{r}$ is not just the price for workshared mail, it is also the access price charged to $E$ to deliver its mail in the rural area.

To write the cost and profit functions of the operators we have to distinguish between two regimes: access (subscript $a c$ ) and bypass (subscript $b p$ ) in the urban area (recall that there is no bypass in the rural area). Furthermore, we use uppercase letters for (total) market demand levels.

\section{Access}

The cost of operator $I$ is given by

$C_{a c}^{I}=\left(c_{1}+c_{2}^{u}\right) X^{u}+\left(c_{1}+c_{2}^{r}\right) X^{r}+c_{2}^{u}\left(Z_{I}^{u}+Y^{u}+Z_{E}^{u}\right)+c_{2}^{r}\left(Z_{I}^{r}+Y^{r}+Z_{E}^{r}\right)+F+F^{u}+F^{r}$,

while for operator $E$ we have

$$
C_{a c}^{E}=k_{E} Y^{u}+a^{u}\left(Y^{u}+Z_{E}^{u}\right)+k_{E} Y^{r}+a^{r}\left(Y^{r}+Z_{E}^{r}\right)+\Phi .
$$

For the profits, we have respectively:

$$
\begin{aligned}
& \pi_{a c}^{I}=p_{x}\left(X^{u}+X^{r}\right)+p_{z}^{u} Z_{I}^{u}+a^{u}\left(Y^{u}+Z_{E}^{u}\right)+p_{z}^{r} Z_{I}^{r}+a^{r}\left(Y^{r}+Z_{E}^{r}\right)-C_{a c}^{I}, \\
& \pi_{a c}^{E}=q_{y}^{u} Y^{u}+q_{y}^{r} Y^{r}+q_{z}^{u} Z_{E}^{u}+q_{z}^{r} Z_{E}^{r}-C_{a c}^{E} .
\end{aligned}
$$

\section{Bypass}

Under (urban) bypass the following expressions apply :

$$
\begin{aligned}
C_{b p}^{I} & =\left(c_{1}+c_{2}^{u}\right) X^{u}+\left(c_{1}+c_{2}^{r}\right) X^{r}+c_{2}^{u} Z_{I}^{u}+c_{2}^{r}\left(Z_{I}^{r}+Y^{r}+Z_{E}^{r}\right)+F+F^{u}+F^{r},(7) \\
C_{b p}^{E} & =k_{E} Y^{u}+c_{2}^{E}\left(Y^{u}+Z_{E}^{u}\right)+k_{E} Y^{r}+a^{r}\left(Y^{r}+Z_{E}^{r}\right)+\Phi+\Phi^{u} \\
\pi_{b p}^{I} & =p_{x}\left(X^{u}+X^{r}\right)+p_{z}^{u} Z_{I}^{u}+p_{z}^{r} Z_{I}^{r}+a^{r}\left(Y^{r}+Z_{E}^{r}\right)-C_{b p}^{I} \\
\pi_{b p}^{E} & =q_{y}^{u} Y^{u}+q_{y}^{r} Y^{r}+q_{z}^{u} Z_{E}^{u}+q_{z}^{r} Z_{E}^{r}-C_{b p}^{E} .
\end{aligned}
$$


Equations (3)-(6) on the one hand and (7)-(10) on the other hand have of course a very similar structure. The only differences are due to the way $Z_{E}^{u}$ and $Y^{u}$ are processed. Under access $Z_{E}^{u}$ and $Y^{u}$ appear in the incumbent's profits both on the cost and on the revenue side. Their marginal cost to the entrant is given by $a^{u}$ (see also Table 1). Under bypass, neither $Z_{E}^{u}$ nor $Y^{u}$ do at all appear in the incumbents profits. Their marginal cost to the entrant is $c_{2}^{u}$ and the entrant incurs an extra fixed cost $\Phi^{u}$.

\subsection{Pricing of the entrant}

We assume that the entrant realizes exogenously given markups: $\beta_{z}^{u}, \beta_{z}^{r}, \beta_{y}^{u}$ and $\beta_{y}^{r}$ that may differ according to the area and/or the product. Observe that the case where all or some of the markups are equal is not ruled out.

In regime $a c$, prices of $E$ are thus given by:

$$
\begin{aligned}
q_{y}^{u} & =\left(a^{u}+k^{E}\right)\left(1+\beta_{y}^{u}\right), \\
q_{y}^{r} & =\left(a^{r}+k^{E}\right)\left(1+\beta_{y}^{r}\right), \\
q_{z}^{u} & =a^{u}\left(1+\beta_{z}^{u}\right), \\
q_{z}^{r} & =a^{r}\left(1+\beta_{z}^{r}\right) .
\end{aligned}
$$

We assume that in this regime we have $Z_{E}^{u}=Z_{E}^{r}=0$. This assumption appears to be particularly compelling when $p_{z}^{i}=a^{i}$.

In regime $b p$, prices of $E$ in area $u$ are given by:

$$
\begin{aligned}
q_{y}^{u} & =\left(c_{2}^{E}+k^{E}\right)\left(1+\beta_{y}^{u}\right), \\
q_{z}^{u} & =c_{2}^{E}\left(1+\beta_{z}^{u}\right) .
\end{aligned}
$$

Prices in area $r$ are the same as in regime $a c$ and continue to be given by (12) and (14). Following the approach adopted in regime $a c$, we also assume $Z_{E}^{r}=0$ under regime $b p$. In essence this amounts to assuming that the entrant's workshared product is offered only in $u$ and only under bypass.

The constant markup assumption is made for simplicity. It is the simplest framework compatible with the existence of fixed costs for the entrant. Alternatively one could 
assume that the entrant's prices are given by Bertrand type reaction functions; see Billette de Villemeur et al. (2003b). ${ }^{11}$ For instance, we would have

$$
q_{y}^{r}=q_{y}^{r}\left(a^{r}, p_{x}\right)
$$

i.e., the entrant's price (best reply) is a function of the access charge (which determines its cost) but also of the incumbents price. This shows that our specification involves two different restrictions. First, that $\partial q_{y}^{r} / \partial a^{r}$ is constant and, second, that we neglect the strategic interaction through which the incumbents price affects the entrant's pricing policy. One can easily check that the first restriction has no impact on the results we provide; in particular, the optimal pricing rules remain valid. ${ }^{12}$ Accounting for the effect of $p_{x}$ on the other hand would complicate the expressions (there would be extra terms which we implicitly assume to be of second order).

An alternative way to rationalize the constant markup assumption is to assume that the entrant's demand functions have constant price elasticity. In that case (and given that marginal cost is constant) the Bertrand reaction function of the entrant (17) reduces to a simple constant markup rule. ${ }^{13}$

To simplify the subsequent expressions it is convenient to define market demand functions $X^{i}\left(p_{x}, p_{z}^{i}, q_{y}^{i}, q_{z}^{i}\right)$ and $Z_{I}^{i}\left(p_{x}, p_{z}^{i}, q_{y}^{i}, q_{z}^{i}\right)$ for the incumbent and $Y^{i}\left(p_{x}, p_{z}^{i}, q_{y}^{i}, q_{z}^{i}\right)$ and $Z_{E}^{i}\left(p_{x}, p_{z}^{i}, q_{y}^{i}, q_{z}^{i}\right)$ for the entrant $(i=u, r) .{ }^{14}$

\subsection{Bypass and entry decisions}

The entrant will bypass if

$$
\begin{aligned}
& \beta_{y}^{u}\left(a^{u}+k^{E}\right) Y^{u}\left[p_{x}, p_{z}^{u},\left(a^{u}+k^{E}\right)\left(1+\beta_{y}^{u}\right), a^{u}\left(1+\beta_{z}^{u}\right)\right]< \\
& \quad \beta_{y}^{u}\left(c_{2}^{E}+k^{E}\right) Y^{u}\left[p_{x}, p_{z}^{u},\left(c_{2}^{E}+k^{E}\right)\left(1+\beta_{y}^{u}\right), c_{2}^{E}\left(1+\beta_{z}^{u}\right)\right] \\
& \quad+\beta_{z}^{u} c_{2}^{E} Z_{E}^{u}\left[p_{x}, p_{z}^{u},\left(c_{2}^{E}+k^{E}\right)\left(1+\beta_{y}^{u}\right), c_{2}^{E}\left(1+\beta_{z}^{u}\right)\right]-\Phi^{u} .
\end{aligned}
$$

\footnotetext{
${ }^{11}$ In other words, we have a leader follower setting.

${ }^{12}$ Some redefinitions of elasticities are required, though.

${ }^{13}$ This is a commonly used shortcut in monopolistic competition model; see Tirole (1988), ch. 7. Observe that Crew and Kleindorfer (2006) also work with a specification that involves a constant markup. However, they adjust the markup so that fixed costs are just covered. We simply assume that markups are exogenous but argue that this may be consistent with profit maximization.

${ }^{14}$ The formal definitions are straightforward but somewhat tedious. They are contained in a technical appendix available on the authors' website at www.idei.fr.
} 
The LHS of this expression is the profit of operator $E$ in area $u$ if he uses the incumbent's network to deliver the mail. ${ }^{15}$ Recall that the operator applies a constant markup. To determine the profit we can thus multiply unit costs by the markup and multiply by the quantity. The RHS is the profit when he uses his own network (and thus incurs the extra fixed cost $\left.\Phi^{u}\right)$.

Assuming that the LHS of this expression is decreasing in $a^{u}$, there exists a critical value of the access charge, $\widetilde{a}^{u}$ such that the operator bypasses whenever $a^{u}>\widetilde{a}^{u}$ and uses I's delivery network otherwise. This critical level is of course increasing in $\Phi^{u}$. Note that when the constraint $a^{u}=p_{z}^{u}$ is imposed this becomes more complicated.

\section{$3 \quad$ Results with general demand}

The model we have introduced is rather complicated. In particular, uniform pricing of $x$ and (possibly) $z$, will lead to complicated expressions. To keep the model as simple as possible we shall concentrate on two special (and extreme) cases. We shall successively assume $\alpha=0$ (all addressees are in area $r$ ) and then $\alpha=1$ (all addressees are in area $u$ ). The first setting $\alpha=0$ yields the no bypass case; the entrant's products are delivered by the incumbent. This is similar to Billette de Villemeur et al. (2005), except that the entrant's pricing strategy is different (constant markup rather than competitive fringe). We assume $p_{z}=a$ so that worksharing customers and competitors pay the same price for delivery. The second setting, $\alpha=1$ is the "full bypass" case where all the entrant's products are delivered through its own network. The access price only applies to the worksharing customers.

In either case we make other, more specific assumptions which will be discussed below. The main advantage of looking at these extreme cases is that we do not have to worry about uniform pricing. Furthermore, since we have a pure access and a pure bypass regime, we can try to compare the pricing rules to assess the impact of bypass on access prices.

Before proceeding let us point out that while the two cases we consider are admittedly restrictive their analysis has more far-reaching implications. This is because the

\footnotetext{
${ }^{15}$ Making use of the assumption that $Z_{E}^{u}=0$ under access.
} 
results remain valid when $0<\alpha<1$, as long as there is no uniform pricing. To be more precise, the expressions for area $r$ are then simply those we obtain for $\alpha=0$, while those for $u$ correspond to the solution with $\alpha=1$. With uniform pricing, on the other hand, expressions would be significantly more complex. As the FOCs would then be convex combinations of the conditions we obtain, an analytical study of the pricing rules would no longer appear to be possible. ${ }^{16}$

\subsection{The case $\alpha=0$}

By setting $\alpha=0$, we assume that all mail is to be delivered in the rural area where, by assumption, there is no bypass. Furthermore, we impose $a^{r}=p_{z}^{r}$ and assume $Z_{E}^{r}=0$ : under access the entrant does not offer $z$. This is necessarily true when for the workshared mail, $I$ and E's products are sufficiently close substitutes to obtain $Z_{E}^{r}\left(q_{z}^{r}, p_{z}^{r}\right)=0$ if $q_{z}^{r}>a^{r}=p_{z}^{r}$. In this case we return to the setting of Billette de Villemeur et al. (2005) and the pricing rules can be obtained from the expression provided there by redefining $q_{y}^{r}\left(p_{z}^{r}\right)$ according to $(12) .{ }^{17}$ This yields

$$
\begin{aligned}
\frac{p_{x}-\left(c_{1}+c_{2}^{r}\right)}{p_{x}} & =\frac{\lambda}{1+\lambda} \frac{1}{\widehat{\sigma}_{X}^{r}} \\
\frac{p_{z}^{r}-c_{2}^{r}}{p_{z}} & =\frac{\lambda}{1+\lambda} \frac{1}{\widehat{\sigma}_{Y+Z_{I}}^{r}}
\end{aligned}
$$

where $\widehat{\sigma}_{X}^{r}$ and $\widehat{\sigma}_{Y+Z_{I}}^{r}$ are the superelasticities of $\widetilde{X}^{r}\left(p_{x}^{r}, p_{z}^{r}\right)=X^{r}\left[p_{x}^{r}, p_{z}^{r}, q_{y}^{r}\left(p_{z}^{r}\right)\right]$ and $\widetilde{Y}^{r}\left(p_{x}^{r}, p_{z}^{r}\right)+\widetilde{Z}_{I}^{r}\left(p_{x}^{r}, p_{z}^{r}\right)=Y^{r}\left[p_{x}^{r}, p_{z}^{r}, q_{y}^{r}\left(p_{z}^{r}\right)\right]+Z_{I}^{r}\left[p_{x}^{r}, p_{z}^{r}, q_{y}^{r}\left(p_{z}^{r}\right)\right]$ respectively. ${ }^{18}$

Consequently, the access/workshared price $p_{z}=a$ is determined by the usual inverse superelasticity rule. Since the price pertains to two products $Y$ and $Z_{I}$ (which have the same cost for $I$, but different demands) the superelasticity is that of the sum of these demands and we can think about $Y+Z_{I}=\widetilde{Y}^{r}\left(p_{x}^{r}, p_{z}^{r}\right)+\widetilde{Z}_{I}^{r}\left(p_{x}^{r}, p_{z}^{r}\right)$ as aggregate demand for "access" (or rather access cum worksharing).

\footnotetext{
${ }^{16}$ Even with linear demand functions, one would have to resort to numerical simulations.

${ }^{17}$ Billette de Villemeur et al. (2005) consider a competitive fringe which amount to setting all the $\beta$ 's to zero. The current setting is thus a generalization of Billette de Villemeur et al. (2005) but the expression are derived exactly along the same lines (and interestingly change very little essentially because the markup is a multiplicative factor which cancels out in the various elasticities.)

${ }^{18}$ Recall that the definition of $\widetilde{X}^{r}\left(p_{x}^{r}, p_{z}^{r}\right)$ accounts for the induced price variations of the entrant; see Billette de Villemeur et al. (2005), p. 149.
} 


\subsection{The case $\alpha=1$}

Assume now that all mail is to be delivered in the urban area where the entrant has his own delivery network. Access is now only provided to worksharing customers. For the time being, we do not analyze the bypass decision of the entrant; we simply assume that bypass occurs at the equilibrium. Put differently, all the expressions here are derived conditional on there being bypass. Doing this, we neglect two aspects. First, the optimal $p_{z}$ in the access regime (determined according to (19) for a marginal cost of $c_{2}^{u}$ ) may be such that bypass is not interesting anyway (for instance, because $\Phi^{u}$ is large). Second, even when this is not true, the incumbent might find it desirable to lower its price to discourage bypass. We shall revisit this issue below.

The entrant's prices are determined by (15) and (16). Consequently, they are given and do not depend on the incumbent's prices. This implies that the structure of operator I's pricing problem is exactly the same as in the monopoly case studied by Billette de Villemeur et al. (2003a). There are terms in the objective function which were not present in the monopoly case (e.g. the profit of $E$ ) but these are constant when it comes to determining $I$ 's price structure. The level and elasticity of demand for $X$ and $Z$ will of course differ from the monopoly case, but like in that situation these are effectively only functions of $p_{x}$ and $p_{z}$ (the other arguments being constant).

It thus follows that the optimal pricing rules are given by expression (15) and (16) of Billette de Villemeur et al. (2003a) so that we have (with the notation of the current paper)

$$
\begin{aligned}
\frac{p_{x}-\left(c_{1}+c_{2}^{u}\right)}{p_{x}} & =\frac{\lambda}{1+\lambda} \frac{1}{\sigma_{X}^{u}}=\frac{\lambda}{1+\lambda} \frac{1}{\widehat{\sigma}_{X}^{u}}, \\
\frac{p_{z}^{u}-c_{2}^{u}}{p_{z}^{u}} & =\frac{\lambda}{1+\lambda} \frac{1}{\sigma_{Z_{I}}^{u}}=\frac{\lambda}{1+\lambda} \frac{1}{\widehat{\sigma}_{Z_{I}}^{u}}
\end{aligned}
$$

where $\sigma_{X}^{u}$ and $\sigma_{Z_{I}}^{u}$ are the superelasticities of $X$ and $Z_{I}$ respectively. Observe that we do not have to distinguish between $X$ and $Z_{I}$ on the one hand and $\widetilde{X}$ and $\widetilde{Z}_{I}$ on the other hand because the indirect effect through the price of the other operator does not occur here. Consequently, we have $\sigma_{X}^{u}=\widehat{\sigma}_{X}^{u}$ and $\sigma_{Z_{I}}^{u}=\widehat{\sigma}_{Z_{I}}^{u}$ so that $\widehat{\sigma}$ 's are not needed here; they are only used for reasons of symmetry with expression (18) and (19). 
Let us now consider the implications of (18)-(21) for $p_{z}$ under access and bypass. We set $c_{2}^{u}=c_{2}^{r}=c_{2}$ (which makes sense here) and then have to compare $\widehat{\sigma}_{Z_{I}}^{u}$ and $\widehat{\sigma}_{Y+Z_{I}}^{r}$ or dropping the index (because in each case we are considering the entire set of addressees) $\widehat{\sigma}_{Z_{I}}$ and $\widehat{\sigma}_{Y+Z_{I}}$. On purely theoretical grounds this comparison does not appear to be unambiguous; to be more precise it is an empirical issue. The following elements appear to be relevant. First, in $\widehat{\sigma}_{Z_{I}}$, we have a single product whereas $\widehat{\sigma}_{Y+Z_{I}}$ includes two products. It is not clear how this affects the comparison. Second, and more significantly, the content of $Z_{I}$ is not the same in the two expressions. Under bypass, $Z_{I}$ has a direct competitor and thus substitute (namely $Z_{E}$ ). Under access, on the other hand, there is no alternative to $Z_{I}$; even when the customer patronizes $E$, the mail will eventually be delivered by $I$. For this reason, we can expect the elasticity of $Z$ to be significantly higher under bypass. Third, the value of $\lambda$ is likely to differ between the two cases and possibly in ways that offset the impact of the higher elasticities of $Z$ under bypass.

Summing up, it appears possible to make a case for $p_{z}$ to be lower under bypass (even when "strategic", bypass preventing behavior is not accounted for). Introducing such strategic behavior can of course only reinforce this conclusion.

\section{Access vs. bypass with perfect substitutes}

Let us now assume that the entrant's and the incumbent's products are perfect substitutes. More precisely we assume that $x$ and $y$ are perfect substitutes, so that the same property applies to $z_{E}$ and $z_{I}$. Once again, we successively study the cases $\alpha=0$ (access) and $\alpha=1$ (full bypass). The first case has essentially already been studied in Billette de Villemeur et al. (2004), except that we have a markup for the entrant rather than the competitive fringe.

\subsection{The case $\alpha=0$ (no bypass)}

In this case we have either $q_{y}^{r}>p_{x}$ (Regime $I$ ) or $q_{y}^{r} \leq p_{x}$ (Regime $I I$ ). Recall that product $z_{E}$ is not provided. 


\subsubsection{Regime $I: q_{y}^{r}>p_{x}$}

In this case, the entrant's market share is zero and we return to the monopoly setting of Billette de Villemeur et al. (2003a). The case is excluded by assumption (the entrant has no revenue and cannot cover its fixed cost; anticipating this, he would not enter in the first place).

\subsubsection{Regime $I I: q_{y}^{r} \leq p_{x}$}

To assess if this regime occurs, we have to compare $q_{y}^{r}\left(p_{z}^{r}\right) \geq k^{E}+p_{z}^{r}$ to $p_{x}=\widetilde{k}^{r}+p_{z}^{r}$. A necessary condition for $q_{y}^{r} \leq p_{x}$ is that $k^{E}<\widetilde{k}^{r}=p_{x}-p_{z}^{r}$. In this regime, we have $X^{r}\left(p_{x}, p_{z}^{r}\right)=X_{h}^{r}\left(p_{x}\right)$ : the good $X$ is consumed only by households. The mail of the different firms is split between the entrant $E$ (for the high $k$ ) and the incumbent $I$ (for the low $k$ ) as shown on Figure 2 and we have

$$
\begin{aligned}
Z^{r}\left(p_{z}^{r}\right) & =\int_{\underline{k}}^{q_{y}^{r}-p_{z}^{r}} z_{k}^{r}\left(p_{z}^{r}+k\right) g(k) d k, \\
Y^{r}\left(q_{y}^{r}\right) & =\int_{q_{y}^{r}-p_{z}^{r}}^{\bar{k}} y_{k}^{r}\left(q_{y}^{r}\right) g(k) d k .
\end{aligned}
$$

Note that, since $y$ and $x$ (hence $z$ ) are perfect substitutes, $y_{k}(p)=z_{k}(p)$. As a result, this is "as if" the entry of firm $E$ were allowing firms with $k \geq q_{y}^{r}-p_{z}^{r} \geq k^{E}$ to sort their mail at a cost of only $k^{E}$. Firm $I$ is now facing a set of firms with a distribution of $k$ which is cut from above at the level $q_{y}^{r}-p_{z}^{r}$ and all these firms consume the workshared mail. Note however that this allocation is not necessarily efficient since if the entrant makes a positive markup, it follows that $q_{y}^{r}>k^{E}+a^{r}$ hence even if $a^{r}=p_{z}^{r}$ some firms with $k>k^{E}$ will nevertheless find it profitable to workshare their mail (instead of letting the entrant prepare it).

\subsection{The case $\alpha=1$}

We now turn to the case with full bypass. If the incumbent's products are perfect substitutes, then the demand for a product is positive only if the corresponding operator offers the lowest price. It follows that a firm with a sorting cost $k$ consumes $x_{f}^{u}$ when 


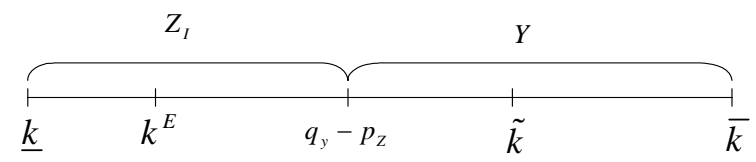

Figure 2: Partition of type $f$ customers in Regime $I I$ under access. Customers with $k<q_{y}-p_{z}$ consume workshared mail (provided by the incumbent). Customers with larger levels of $k$ buy the entrant's end-to-end product $Y$.

the following conditions hold

$$
\begin{aligned}
& p_{x} \leq q_{y}^{u}=\left(c_{2}^{E}+k^{E}\right)\left(1+\beta_{y}^{u}\right) \\
& p_{x} \leq p_{z}^{u}+k \\
& p_{x} \leq q_{z}^{u}+k=c_{2}^{E}\left(1+\beta_{z}^{u}\right)+k .
\end{aligned}
$$

Similarly, it consumes $z_{f}^{u}$ when the following conditions are satisfied

$$
\begin{aligned}
& p_{z}^{u}+k \leq q_{z}^{u}+k=c_{2}^{E}\left(1+\beta_{z}^{u}\right)+k, \\
& p_{z}^{u}+k \leq p_{x} \\
& p_{z}^{u}+k \leq q_{y}^{u}=\left(c_{2}^{E}+k^{E}\right)\left(1+\beta_{y}^{u}\right),
\end{aligned}
$$

For given levels of $q_{y}^{u}, q_{z}^{u}$ we then obtain 4 regimes, as illustrated in Figure 3.

Regime $I$ : the entrant is more expensive for all products $p_{x}<q_{y}^{u}$ and $p_{z}^{u}<q_{z}^{u}$. In this case, the entrant does not enter. This solution is equivalent to Regime $I$ in the 


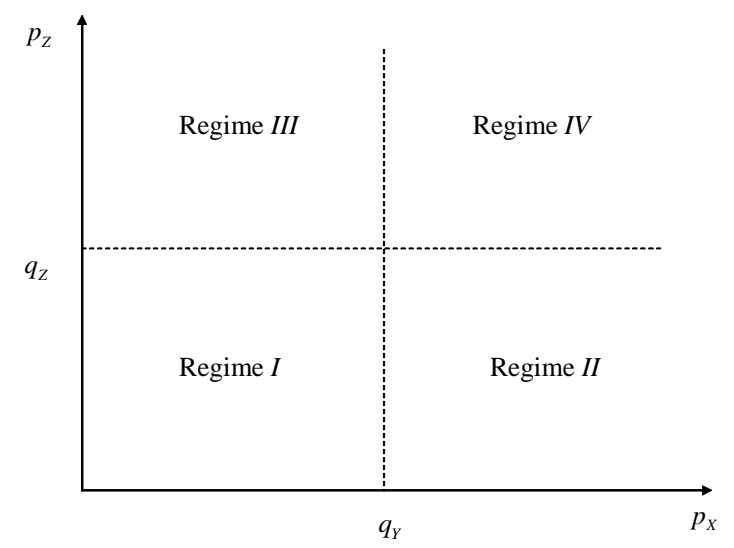

Figure 3: Regimes in the bypass case.

no bypass case. Once again, it is excluded by assumption (the entrant has no revenue and cannot cover its fixed cost; anticipating this, he would not enter in the first place).

Regime $I I$ : the entrant is cheaper for end-to-end mail only: $p_{x}>q_{y}^{u}$ but $p_{z}^{u}<q_{z}^{u}$. In this case, the demand for commodity $X$ reduces to the one of households $X_{h}^{u}\left(p_{x}\right)$. Firms workshare their mail to the incumbent (consume $z_{I}$ ) when $p_{z}+k \leq q_{y}^{u}$; otherwise, they consume $Y$; see Figure 4. Observe that this regime is similar to Regime $I I$ in the no bypass case discussed in subsection 4.1.2. The difference is that the entrant's end-to-end product is no longer delivered by the incumbent. This is to a large extent an artifact of our assumption that there is effectively bypass. With $p_{z}^{u}<q_{z}^{u}$ this is, however, not likely to occur when the bypass decision is endogenous. In that case we would thus effectively return to Regime $I I$ in the access case.

The price $p_{x}$ is the same as in the no bypass case, Regime $I I$ :

$$
\frac{p_{x}-\left(c_{1}+c_{2}^{u}\right)}{p_{x}}=\frac{\lambda}{1+\lambda} \frac{1}{\varepsilon_{X_{h}^{u}}}
$$




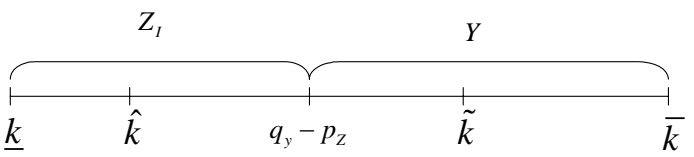

Figure 4: Partition of type $f$ customers in Regime $I I$ under bypass. Customers with $k<q_{y}-p_{z}$ consume workshared mail (provided by the incumbent). Customers with larger levels of $k$ buy the entrant's end-to-end product $Y$.

The demand for $Z^{u}$ writes

$$
Z_{I}^{u}\left(p_{z}^{u}, q_{y}^{u}\right)=\int_{\underline{k}}^{q_{y}^{u}-p_{z}^{u}} z_{k}^{u}\left(p_{z}^{u}+k, k\right) g(k) d k .
$$

Interestingly enough, it does not depend on $p_{x}$. Consequently, the optimal price $p_{z}^{u}$, as defined by the standard Ramsey expression

$$
\frac{p_{z}^{u}-c_{2}^{u}}{p_{z}^{u}}=\frac{\lambda}{1+\lambda} \frac{1}{\varepsilon_{Z_{I}^{u}}}
$$

is also independent from $p_{x}$, for a given level of $\lambda$. The aggregate elasticity $\varepsilon_{Z_{I}^{u}}$ can be decomposed as follows:

$$
\begin{aligned}
\varepsilon_{Z_{I}^{u}} & =\frac{p_{z}^{u}}{Z_{I}^{u}}\left(\int_{\underline{k}}^{q_{y}^{u}-p_{z}^{u}} \frac{-\partial z_{k}^{u}\left(p_{z}^{u}+k, k\right)}{\partial p_{z}^{u}} g(k) d k+z_{k}^{u}\left(q_{y}^{u}\right) g\left(q_{y}^{u}-p_{z}^{u}\right)\right) \\
& =\int_{\underline{k}}^{q_{y}^{u}-p_{z}^{u}} \alpha_{Z_{I}^{u}}^{k} \varepsilon_{k}^{u} d k+\alpha_{Z_{I}^{u}}^{k=q_{y}^{u}}
\end{aligned}
$$

where $\alpha_{Z_{I}^{u}}^{k}=g(k) z_{k}^{u} / Z_{I}^{u}$ is the share of demand by type $k$ firms and $\varepsilon_{k}^{u}$ the price elasticity. Clearly when $q_{y}^{u}$ increases, the interval $\left[\underline{k}, q_{y}^{u}-p_{z}^{u}\right]$ increases incorporating firms with lower elasticities which suggest that the aggregate elasticity will also decrease. As a result, the socially optimal price $p_{z}^{u}$ increases with $q_{y}^{u}$. 
Regime $I I I$ : the entrant is cheaper for workshared mail only: $p_{x}<q_{y}^{u}$ but $p_{z}^{u}>q_{z}^{u}$. For this regime to occur, we have to assume that:

$$
\begin{aligned}
& p_{x}<q_{y}^{u}=\left(1+\beta_{y}^{u}\right)\left(c_{2}^{E}+k^{E}\right), \\
& q_{z}^{u}=c_{2}^{E}\left(1+\beta_{z}^{u}\right)<p_{z}^{u},
\end{aligned}
$$

which is unlikely to arise if the markup of entrants is sufficiently low and their upstream costs are well below those of the incumbent.

Assume nevertheless that both of these inequalities hold. In this case, the demand for the commodity $Z_{I}^{u}$ is equal to zero. As a result, there is no loss on the incumbent's revenue if price $p_{z}$ is reduced to $c_{2}^{u}$. It follows that $q_{z}^{u}=c_{2}^{E}\left(1+\beta_{z}^{u}\right) \leq c_{2}^{u}$ is a necessary condition for Regime $I I I$ to arise.

In this regime, as illustrated by Figure 5, consumers of type $f$ with low levels of $k$ consume $z_{E}$, while those with high $k$ 's consume $x$. The only demand for the incumbent's products is the demand for commodity $X$ :

$$
X^{u}\left(p_{x}, q_{z}^{u}\right)=X_{h}^{u}\left(p_{x}\right)+\int_{p_{x}-q_{z}^{u}}^{\bar{k}} x_{f}^{u}\left(p_{x}, k\right) g(k) d k .
$$

Interestingly enough, it does not depend on $p_{z}$. The price $p_{x}$ is set according to the Ramsey rule

$$
\frac{p_{x}-\left(c_{1}+c_{2}^{u}\right)}{p_{x}}=\frac{\lambda}{1+\lambda} \frac{1}{\varepsilon_{X^{u}}}
$$

and is independent from $p_{z}^{u}$, for a given level of $\lambda$. The aggregate price-elasticity is

$$
\varepsilon_{X^{u}}=\frac{p_{x}}{X^{u}}\left(\frac{-\partial X_{h}^{u}\left(p_{x}\right)}{\partial p_{x}}+\int_{p_{x}-q_{z}^{u}}^{\bar{k}} \frac{-\partial x_{f}^{u}\left(p_{x}, k\right)}{\partial p_{x}} g(k) d k+x_{f}^{u}\left(p_{x}, p_{x}-q_{z}^{u}\right) g\left(p_{x}-q_{z}^{u}\right)\right)
$$

which is likely to increase when $q_{z}^{u}$ decreases. (The "high elasticity consumer" shift to the entrants product). As a result the socially optimal price $p_{x}$ increases with $q_{z}^{u}$.

Observe that, in Regime $I I I$, the incumbent's demand emanates only from households and from firms with high preparation cost (that are likely to be also the customers with a lower volume). Compared to the previous two regimes, the incumbent has thus totally lost a crucial market segment (namely the firms with low preparation cost). Budget balancing would thus "at best" require a rather large price for $X$. Moreover, 


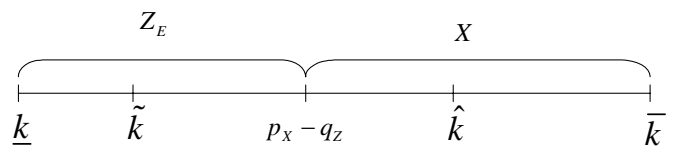

Figure 5: Partition of type $f$ customers in Regime $I I I$ under bypass. Customers with $k<q_{z}-p_{x}$ consume the entrant's workshared mail product. Customers with larger levels of $k$ buy the incumbent's end-to-end product $X$.

since the remaining market segments can be expected to yield a rather low volume, it may well be the case that the highest possible (gross) profit that can be achieved is not sufficient to cover the fixed cost. We shall return to this point below.

Regime $I V$ : the entrant is cheaper for both type of products $p_{x}>q_{y}^{u}$ and $p_{z}^{u}>$ $q_{z}^{u}$. In this case the demand for the incumbent reduces to the demand of households $X^{u}\left(p_{x}, p_{z}^{u}, q_{y}^{u}, q_{z}^{u}\right)=X_{h}^{u}\left(p_{x}\right)$. Compared to Regime $I I I$ an additional segment (namely the firms with high preparation costs) has been lost by the incumbent. With a single category of customers left, it is now even more unlikely that the incumbent would be able to break-even.

When break even is possible the optimal pricing rule is given by

$$
\frac{p_{x}-\left(c_{1}+c_{2}^{u}\right)}{p_{x}}=\frac{\lambda}{1+\lambda} \frac{1}{\varepsilon_{X_{h}^{u}}},
$$

where $\lambda$ adjusts for the firm to be able to break-even. When the fixed cost to be covered equals the (gross) monopoly profit the multiplier $\lambda$ goes to infinity and the incumbent does not differ from a profit-maximizing firm. For higher levels of $F$ no solution exists and the incumbent would not be able to survive.

When do these regimes occur? In Figure 3 and in the subsequent discussion, we have characterized the regimes by conditions that involve endogenous variables (in par- 


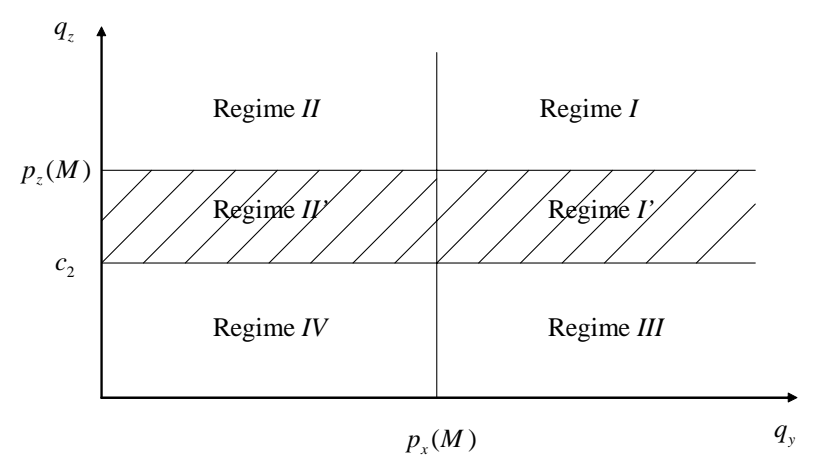

Figure 6: Regimes in the bypass case in the $\left(q_{y}, q_{z}\right)$ space.

ticular $p_{x}$ and $p_{z}$ ). This presentation is most convenient in that it allows us to easily get an intuitive understanding of the implication of the different regimes. However, it does not give us a full characterization of the solution and it does not tell us precisely which exogenous parameter combination result in which regime. To address this question, we use a representation in the $\left(q_{y}, q_{z}\right)$ space (see Figure 6). Recall that under bypass, both of these prices are exogenous and given by equations (15) and (16). In this space, we depict the incumbent's (welfare maximizing) prices under monopoly $p_{x}(M)$ and $p_{z}(M)$. These levels are also well defined and can be considered as exogenous for the question under investigation here. The incumbent's delivery $\operatorname{cost} c_{2}$ is also depicted on the vertical axis. It is plain that when $q_{y}>p_{x}(M)$ and $q_{z}>p_{z}(M)$ we will have Regime $I$, while $q_{y}<p_{x}(M)$ and $q_{z}<c_{2}$ yields Regime $I V$. Similarly, one easily shows that $q_{y}<p_{x}(M)$ along with $q_{z}>p_{z}(M)$ yields Regime $I I$ while $q_{y}>p_{x}(M)$ and $q_{z}<c_{2}$ results in Regime $I I I$. To see this, one has to recall the properties of the different regimes discussed above, while keeping in mind the regimes depend on the incumbent's effective price (and not directly on the hypothetical monopoly price). ${ }^{19}$ The intermediate (transitional) regimes $I^{\prime}$ and $I I^{\prime}$ require a more careful explanation. For

\footnotetext{
${ }^{19}$ For instance, when $q_{y}>p_{x}(M)$ and $q_{z}>p_{z}(M)$ the incumbent can just set the same prices as under monopoly.
} 
instance, when $q_{y}>p_{x}(M)$ along with $p_{z}(M)>q_{z}>c_{2}$, the incumbent would end up in Regime $I I I$ if he were to maintain its high monopoly price (for $z$ ). Clearly it is a better strategy to "undercut" the entrant to retain the industrial mail segment, as long as this yields a price which exceeds the incumbent's delivery $\operatorname{cost} c_{2}$ (and thus a positive markup which contributes towards the financing of fixed costs). This corresponds to the strategic bypass deterring we have not addressed above.

\section{Concluding remarks}

This paper has studied the potential market equilibria in a liberalized postal market where a regulated incumbent (the universal service provider) competes with non regulated entrants. The model accounts for a number of features that are already or may become relevant in the postal sector in most European member states. These include the possibility of access, bypass, consolidation and worksharing. Doing this, we have considered that entrants may offer end-to-end products or concentrate on one of the segments of the network. We have shown that a wide variety of scenarios can emerge depending on the regulatory environment and on the relative cost and demand structures for the incumbent and the entrants' products.

To wrap up and to draw some lessons, let us concentrate on the case where the products offered by incumbent and entrant are perfect substitutes (Section 4). To stress the main contributions of this paper, we shall particularly emphasize the impact of bypass. We have seen that when bypass is possible a number of possible scenarios can arise.

It may be the case that liberalization induces no changes compared to the (regulated) monopoly scenario. This is the case when the (regulated) monopoly prices make entry by any potential operator non profitable (Regime $I$ ). When entry effectively does occur, the new operator may offer cheaper service than the incumbent in some or in all market segments. The most favorable case for the incumbent is when the entrant is cheaper only for end-to-end mail (Regime $I I$ ). This can occur for instance when the entrant is able to sort mail at a lower cost than the incumbent $\left(k^{E} \leq c_{1}\right)$. In this case, the incumbent's end-to-end product is consumed exclusively by households. Firms (and the 
entrant operator in the no bypass case) use the incumbent's delivery services only for the workshared mail. Compared to Regime $I$, the incumbent has lost market share, but it retains probably the most lucrative segment, namely the firms with low preparation costs. Observe that both of these regimes can occur in the access as well as in the bypass case. Even if it is theoretically possible, bypass will not have a significant impact under these two regimes. Consequently, bypass is unlikely to occur in the first place. In other words when the model is extended to account for the bypass decision (as discussed in Subsection 2.4), it is not likely that the entrant will find it profitable to build its own delivery network.

A very different result obtains when the entrant offers cheaper service in delivery. This can occur for instance in relatively dense areas where the delivery costs of the entrant may be well below the (possibly uniform) delivery price charged by the incumbent $\left(c_{2}^{E}<c_{2}^{E}\left(1+\beta_{z}^{u}\right) \leq p_{z}^{u}\right)$. In this case, the incumbent loses the entire pre-sorted mail market (Regime $I I I$ ). It is left with demand emanating only from households and from firms with high preparation cost (that are likely to be also the customers with lower volumes). Since the remaining market segments can be expected to yield a rather low volume, the universal service operator's revenues may no longer be sufficient to cover its fixed cost. Consequently, under Regime $I I I$ the financial viability of the universal service operator is jeopardized.

This would be even more likely in Regime $I V$, where the incumbent is left solely with the demand from households. Volumes are then even smaller than in Regime III and one cannot expect them to provide a sufficient contribution to cover the universal provider's fixed costs. It is not clear how universal service (and specifically service to households) can be guaranteed in this setting. Observe that the two critical regimes require bypass. Absent bypass, the existence of the incumbent does not appear to be seriously threatened, as long of course as access charges are set appropriately.

Throughout the paper, we have implicitly restricted the incumbent's pricing strategies in several ways. The restrictions are meant to account for regulatory constraints that are often imposed in practice. In particular, we have not considered price differentiation (for instance between households and firms) and we have not considered the 
possibility of strategic "bypass deterrence" (setting an access price just below the competitor's delivery cost). When the incumbent's pricing policy is restricted, the entrant may be able to offer services at a lower price only because it is able to differentiate its prices between customers (while the incumbent is subject to uniform or non discriminatory pricing requirements). In that case, allowing the incumbent to differentiate prices as well may improve welfare. More generally, providing the universal service provider with more flexible pricing tools could mitigate the threat that bypass poses to its survival.

\section{References}

[1] Armstrong, M. 2002. "The theory of access pricing and interconnection", in Handbook of Telecommunication, vol 1., edited by M. Cave et al., 295-386.

[2] Billette de Villemeur, E, H. Cremer, B. Roy. and J. Toledano. 2002. "Pricing and worksharing discounts in the postal sector" in Postal and Delivery Services: Delivering on Competition, edited by M. A. Crew and P. R. Kleindorfer, Boston : Kluwer Academic Publishers, 33-48.

[3] Billette de Villemeur, E, H. Cremer, B. Roy. and J. Toledano. 2003a. "Optimal pricing and global price-cap in the postal sector", Journal of Regulatory Economics, 24, 49-62.

[4] Billette de Villemeur, E, H. Cremer, B. Roy. and J. Toledano. 2003b., "Pricing and imperfect competition in the postal sector", mimeo, IDEI, University of Toulouse.

[5] Billette de Villemeur, E, H. Cremer, B. Roy. and J. Toledano. 2004. "Access and (non-)uniform pricing in the postal sector" in Competitive Transformation of the Postal and Delivery Sector, edited by M. A. Crew and P. R. Kleindorfer, Boston: Kluwer Academic Publishers, 43-68.

[6] Billette de Villemeur, E, H. Cremer, B. Roy. and J. Toledano. 2005. "Worksharing, pricing and competition in the postal sector" in Regulatory and Economic Challenges in the Postal and Delivery Sector, edited by M. A. Crew and P. R. Kleindorfer, Boston : Kluwer Academic Publishers, 139-162. 
[7] Crew M. and P. Kleindorfer. 1995. "Pricing in postal service under competitive entry", in Commercialization of Postal and Delivery Services, edited by M. A. Crew and P. R. Kleindorfer, Boston: Kluwer Academic Publishers, 117-136.

[8] Crew M. and P. Kleindorfer. 2006. "The welfare effect of entry and strategies for maintaining the USO in the postal sector", in Progress toward Liberalization of the Postal and Delivery Sector, edited by M. A. Crew and P. R. Kleindorfer, New York : Springer, 3-22.

[9] Laffont, J.J. and J. Tirole. 1996. "Creating competition through interconnection: Theory and practice", Journal of Regulatory Economics, 10, 227-256.

[10] Laffont, J.J. and J. Tirole. 2000. Competition in Telecommunications, MIT Press, Cambridge MA.

[11] Mitchell, R. 1999. Postal worksharing: welfare, technical efficiency, and Pareto optimality, in Emerging Competition in Postal and Delivery Services, edited by M. A. Crew and P. R. Kleindorfer, Boston: Kluwer Academic Publishers.

[12] Panzar, J. 2002. "Reconciling competition, downstream access and universal service in postal markets", in Postal and Delivery Services: Delivering on Competition, edited by M. A. Crew and P. R. Kleindorfer, Boston : Kluwer Academic Publishers.

[13] Panzar, J. 2005. "Combining liberalization and unbundling policies in postal markets", mimeo.

[14] Sherman, R. 2001. "Optimal worksharing discounts", Journal of Regulatory Economics, 19, 81-92.

[15] Tirole, J. 1988. The Theory of Industrial Organization, MIT Press, Cambridge MA. 